\title{
BMJ Open Determinants of inappropriate admission of elderly people in county- level hospitals: a cross-sectional study in rural China
}

\author{
Xiaomei Hu, ${ }^{1,2}$ Hongxia Gao, ${ }^{1,2}$ Yan Zhang, ${ }^{\oplus 1,2}$ Haomiao Li, ${ }^{1,2}$ Dai Su, ${ }^{1,2}$ \\ Jingjing Chang, ${ }^{1,2}$ Di Jiang, ${ }^{1,2}$ Shihan Lei, ${ }^{1,2}$ Yingchun Chen ${ }^{\oplus, 2}$
}

To cite: Hu X, Gao H, Zhang Y, et al. Determinants of inappropriate admission of elderly people in county-level hospitals: a cross-sectional study in rural China. BMJ Open 2019;9:e026443. doi:10.1136/ bmjopen-2018-026443

- Prepublication history and additional material for this paper are available online. To view these files, please visit the journal online (http://dx.doi. org/10.1136/bmjopen-2018026443).

$\mathrm{XH}$ and $\mathrm{HG}$ contributed equally.

Received 7 September 2018 Revised 19 February 2019 Accepted 4 March 2019

Check for updates

(c) Author(s) (or their employer(s)) 2019. Re-use permitted under CC BY-NC. No commercial re-use. See rights and permissions. Published by BMJ.

${ }^{1}$ School of Medicine and Health Management, Tongji Medical

College, Huazhong University of Science and Technology, Wuhan, Hubei, China

${ }^{2}$ Research Center for Rural Health Service, Key Research Institute of Humanities \& Social Sciences of Hubei Province, Wuhan, Hubei, China

Correspondence to

Dr Yingchun Chen;

chenyingchun@hust.edu.cn

\section{ABSTRACT}

Objective The purpose of this paper is to investigate the characteristics and determinants of inappropriate admission to hospital of elderly people in rural China. Design A cross-sectional study of a comparison between the elderly and non-elderly groups of people.

Setting The survey was conducted on the largest countylevel general hospitals in four counties in central and western China.

Participants A total of 652 rural patients admitted in hospitals were surveyed, who were divided into two groups according to age: elderly group $(n=230$, age $\geq 60$ years) and non-elderly group ( $n=422$, age $<60$ years).

Primary measures The Chinese version of the appropriateness evaluation protocol was used to evaluate the inappropriate admission rates. The interactive regression models based on the relationship of age (elderly and non-elderly) with other factors and binary logistic regression models were used in the analysis of the specific factors and determinants of the inappropriate admission of elderly people.

Result The inappropriate admission rate for the rural elderly was $30 \%$, which was lower than that of the nonelderly people (40.8\%). Compared with the non-elderly group, women in the elderly group $(\mathrm{OR}=0.33,95 \% \mathrm{Cl} 0.15$ to 0.73 ) had a lower incidence of inappropriate admission, and elderly people with chronic diseases $(\mathrm{OR}=3.33,95 \% \mathrm{Cl}$ 1.23 to 9.04 ) were more prone to being inappropriately admitted than non-elderly people with chronic diseases. The binary logistic regression analysis shows that county, age $(\mathrm{OR}=0.94 .95 \% \mathrm{Cl} 0.90$ to 0.99$)$, gender $(\mathrm{OR}=0.49$, $95 \% \mathrm{Cl} 0.25$ to 2.98 ), department and response to doctor's admission request were the determinants of the inappropriate admission of elderly patients.

Conclusion The inappropriate admission rate of elderly people in rural China was high. We found that gender and chronic disease are the specific factors that were specific to non-elderly people. County, age, gender, department and response to a doctor's admission request had substantial influence on the inappropriate admission of the elderly in rural China.

\section{INTRODUCTION}

Ageing is a severe challenge facing the Chinese society today. At the end of 2017, China's
Strengths and limitation of this study

To the best of our knowledge, this is the first indepth study of inappropriate admission of elderly people in rural areas in China.

- The inclusion of non-elderly people for comparative analysis emphasised the specificity of the inappropriate hospital admission of elderly people.

- In the search for determinants of inappropriate admission of the elderly, social demographics, objective admission status and subjective cognitive status were all included.

- This cross-sectional study design cannot determine the causal relationship between inappropriate hospital admission of elderly people and related factors.

- The appropriateness evaluation protocol applicable to the entire population may not be the best tool for evaluating inappropriate admission of elderly people.

elderly population aged 60 years and above was 241 million, accounting for $17.3 \%$ of the total population. ${ }^{1}$ In recent years, the rate of hospitalisation for the elderly in rural China has increased from $12.7 \%$ in 2003 to $21.5 \%$ in $2013,{ }^{2}$ which suggests that the utilisation of health services for the rural elderly population in China has increased sharply. And the inappropriate admission rate of people over 60 years in rural China has reached 23.2\%. ${ }^{3}$ The inappropriateness of the elderly's health demand has become a major concern in the supply of health services in China.

Inappropriate admission refers to unnecessary hospitalisation of patients, ${ }^{45}$ which is one of the main forms of excessive demand for hospitalisation services. ${ }^{6}$ Some special national conditions in China determine that the reason for inappropriate admission of elderly people in rural China differs from that in other countries. On the one hand, primary healthcare is backward in rural China, especially the family doctor system, 
which remains far from perfect. Hence, elderly patients lack the correct guidance for their first visit although their cognitive levels decrease with age. ${ }^{7}$ On the other hand, China's institutional endowment system remains imperfect, and hospitalisation services have become the main source of nursing services for the elderly who suffer from chronic diseases and poor health. ${ }^{8}$ Both of these aspects are related to the inappropriate admission behaviour of the elderly. In recent years, the Chinese government has adopted various reform measures that are aimed at guiding patients seeking correct medical care; these measures include the implementation of basic medical insurance payment reform, ${ }^{9}$ hierarchical medical system and family doctor contracted services. However, inappropriate inpatient service utilisation for the elderly remains a problem in rural China.

Inappropriate admission wastes health resources and further increases the economic burden of the elderly in rural areas. It also increases the risk of hospital-acquired infections in elderly people, thereby compromising patients' safety and health. ${ }^{10}{ }^{11}$ Therefore, identifying the determinants of inappropriate hospitalisation is a useful approach for the formulation of targeted interventions that can reduce inappropriate hospital admission rates and improve the efficiencies and qualities of hospitalisation services.

The international study on the inappropriate admission of the elderly is mainly focused on the evaluation of the rate, characteristics and impact factors of inappropriate admission in hospitals. Influencing factors are mainly considered from the perspective of individual characteristics and suppliers. The comprehensive consideration of supply and demand factors is often undermined in most studies. The analysis of individual characteristics generally focuses on diseases and severity of illness in hospitalised elderly patients. Gamper et $a l^{12}$ found that elderly people with chronic diseases are more likely to be inappropriately admitted. Menand $e t a l^{13}$ found that the severity of illness and mention of a cardiac disease are associated with inappropriate admission of people aged 80 years. The analysis of influencing factors from the supplier side mainly focuses on the decision-making of medical service providers and primary healthcare. Bianco et $a l^{14}$ showed that the inappropriate admission of elderly people over 65 years old in southern Italy is mainly influenced by conservative admission treatment decision, which is made by doctors or hospital managers to avoid the risk of medical accidents. Mytton et $a l^{15}$ found that, in the UK, the inappropriate admission of elderly people to the emergency departments of hospitals depends on the quality of doctor admission decisions and the capacities of community health services. Presently, research on inappropriate admission of the elderly in China and the only study covered just the descriptive analysis phase, and the effect of the factors remains unclear. We assume that the appropriateness of admission of elderly people is influenced by both the supply and the demand.
Therefore, we aim to identify the rate and determinants of inappropriate admission of elderly people to hospitals in rural China from the perspective of supply and demand, and to provide targeted strategies for the intervention and control of the hospitalisation behaviours of elderly people.

\section{METHODS}

\section{Setting and participants}

This cross-sectional study was conducted in 2017, and cluster random sampling was adopted. Four counties in central and western China, namely, Dingyuan in Anhui Province, Huining and Weiyuan in Gansu Province and Yilong in Sichuan Province, were selected as sample counties. The counties in eastern China were not included because most counties are shifting to the urban district level through rapid economic and social development. The largest county-level general hospital in each county was surveyed, and the respondents were hospitalised patients in rural households who were grouped into the elderly group (aged 60 years and older) and non-elderly group (under 60 years old).

\section{Sampling and data collection}

For the calculation of sample size, it is estimated that the inappropriate admission rate $P$ in rural areas is $25 \%$, the absolute tolerance $\delta=0.035$ and the confidence level is $95 \%(\alpha=0.05)$ :

$$
N=Z_{\alpha / 2}^{2} P(1-P) / \delta^{2}=1.96^{2} 0.25(1-0.25) / 0.035^{2}=588
$$

Through a cluster sampling method, 170 rural patients in each county hospital were randomly selected. The responses were obtained through the use of questionnaire surveys, and their medical records were collected after they were discharged. To ensure the integrity of the data for each indicator, we excluded the unqualified sample records. Of the 680 records collected, 652 valid records were finally retained, including 230 for the elderly and 422 for the non-elderly.

\section{Measurement}

With the consent of the hospital and the patients, a structured questionnaire survey was used, which included inquiries about demographic characteristics and subjective cognitive indicators. Their medical records were extracted for the collection of objective admission statuses and evaluation of the appropriateness of admission.

\section{Variables}

The outcome variable was the appropriateness of admission to the hospital, with appropriate admission $=0$ and inappropriate admission $=1$.

The independent variables mainly include demographic characteristics (eg, county, gender and age), subjective cognitive status (eg, response to doctor's admission request [RTDAR], disease severity, affordability of hospitalisation costs, comparison of inpatient and 
outpatient reimbursement [CORR] and hospitalisation convenience) and objective admission status (eg, department, disease, health status and diagnosis of chronic diseases $[\mathrm{DOCD}])$. Among the above variables, gender, age, subjective cognitive status, disease, health status and DOCD are from the demand's perspective, while county, RTDAR, affordability of hospitalisation costs, CORR, hospitalisation convenience, department and DOCD can also directly or indirectly reflect the influence of the supplier on the inappropriate admission of the elderly.

\section{Records appropriateness evaluation}

The appropriateness evaluation protocol $(\mathrm{AEP})^{16}$ is widely used in the evaluation of the appropriateness of admission. Yingchun Chen ${ }^{17}$ developed rural appropriateness evaluation protocol (R-AEP), which is suitable for the appropriateness of admission to hospitals operating in rural China (online additional file 1 for details).

The R-AEP criteria were used in the evaluation of the appropriateness of admission in 652 cases. The evaluation was performed by professionally trained personnel. These highly trained personnel were professionals in health policy research, all of whom received $\mathrm{PhD}$ degrees and committed to making fair judgements on records in a strictly standard manner. The R-AEP criteria were based on the value of the medical record. Therefore, all R-AEP-related indicators were extracted, and their actual values were compared with standard values. The record for actual indicator values that match with the standard value was considered appropriate. Medical records with all relevant values that did not meet the AEP criteria were considered as inappropriate admission. The specific case evaluation process was as follows: (1) Evaluating each medical record by two experts. (2) Comparing the consistency of the results of the two experts. (3) Cases with inconsistent results from two experts were sent to a third party and were evaluated by a third party. (4) Comparing the results of admission appropriateness made by a third party with the previous results and making the final judgement about appropriateness of patient admission.

\section{Statistics analysis}

The demographic characteristics, subjective cognitive status and objective admission status were processed via Epidata V.3.2. Diseases were categorised using the International Classification of Diseases 10th revision.

IBM SPSS Statistics V.22.0 was used for statistical analysis. The $t$ test and Pearson's $\chi^{2}$ test were used in the analysis of the distribution characteristics of inappropriate admission of the elderly. For the 652 respondents, the interactive logistic regression model based on the relationship of age (elderly and non-elderly) with other factors was adopted for the analysis of the specific factors affecting the inappropriate admission of elderly people in comparison with those affecting the inappropriate admission of non-elderly people. Taking the appropriateness of admission as the dependent variable, we directly included the main effect of the independent variable to the regression model, and the interaction effect was screened into the model by stepwise regression. Finally, the model was formed.

$$
\begin{aligned}
\text { Logit }(P)= & \beta_{0}+\beta_{1} \text { County }+\beta_{2} \text { Age }+\beta_{3} \text { Gender } \\
& +\beta_{4} \text { Disease }+\beta_{5} \text { Department }+\beta_{6} \text { DOCD } \\
& +\beta_{7} \text { Health status }+\beta_{8} \text { RTDAR }+\beta_{9} \text { Severity } \\
& +\beta_{10} \text { CORR }+\beta_{11} \text { Affordability } \\
& +\beta_{12} \text { Hospitalization convenience } \\
& +\beta_{13} \text { Age } \times \text { Gender }+\beta_{14} \text { Age } \times \text { DOCD }+\varepsilon
\end{aligned}
$$

For 230 elderly respondents, binary logistic regression analysis was used for the identification of determinants of inappropriate admission of the elderly. The patient admission appropriateness was the dependent variable, and the independent variable was selected using stepwise regression. The regression model is as follows:

$$
\begin{aligned}
\text { Logit }(P)= & \beta_{0}+\beta_{1} \text { County }+\beta_{2} \text { Age }+\beta_{3} \text { Gender } \\
& +\beta_{4} \text { Department }+ \text { bet }_{5} \text { RTDAR }+\beta_{6} \text { Affordability }+\varepsilon
\end{aligned}
$$

\section{Patient and public involvement}

Patients were not involved in the development of the study. The results were applicable for publication in a peer-reviewed journal. There is no plan to specifically disseminate the findings to study participants.

\section{Research ethics}

Patient information was not disclosed before the analysis.

\section{RESULTS}

\section{Selected characteristics of the study population}

The study included 652 admitted patients, of which 230 were elderly patients (average age: 70.73 years, female: $52.6 \%$ ) and 422 were non-elderly patients (average age: 28.33 years, female: $49.3 \%$ ). The sampling rates in each hospital were $0.53 \%$ in Yilong (samplers in annual hospitalisations in 2017: 157 in 29 461), 0.54\% in Huining (153 in 28 101), $0.28 \%$ in Dingyuan (176 in 62 096) and $1.41 \%$ in Weiyuan (166 in 11 734). The selected characteristics of the study population are shown in tables 1 and 2. Most of the elderly patients were hospitalised in internal medicine $(79.1 \%)$ and were admitted because of circulatory diseases $(43.9 \%)$. Only $43.9 \%$ of the elderly patients were in good health, and the majority of the patients had chronic diseases $(74.3 \%)$. Older patients had a higher expectation of admission and always felt that their respective diseases were serious. The number of elderly patients who considered the reimbursement for inpatients was higher than that for outpatients accounted for only $43.9 \%$ of the elderly respondents. Only $32.2 \%$ of elderly patients thought that they can fully pay for the hospitalisation, and $49.6 \%$ considered that hospitalisation is inconvenient.

Of the 230 elderly patients surveyed, 69 were inappropriately admitted, at a rate of $30 \%$, which was lower than that of non-elderly patients $(40.8 \%)$. The inappropriate admission rate of elderly people in Dingyuan was the highest $(51.8 \%)$, whereas the lowest was in Yilong 
$(14.5 \%)$; the difference between the four counties was statistically significant $\left(\chi^{2}=222.8, \mathrm{p}<0.001\right)$.

\section{Characteristics of inappropriate admission of the elderly}

The objective admission indicators of elderly patients (table 1) show no significant difference in the distribution of admission appropriateness among gender, age, department, disease, health status and diagnosis of chronic diseases. For the non-elderly group, a younger age corresponds to higher inappropriate admission rate $(\mathrm{p}<0.001)$. Moreover, paediatric patients with the highest inappropriate admission rate $\left(\chi^{2}=255.742, \mathrm{p}<0.001\right)$ and patients with chronic diseases are more likely to be inappropriately admitted $\left(\chi^{2}=9.092, \mathrm{p}=0.003\right)$.

The characteristics of inappropriate admission under patients' subjective perceptions is indicated in table 2 . The inappropriate admission rate of elderly patients varies with differences in reimbursement for inpatients and outpatients $\left(\chi^{2}=211.8, \mathrm{p}=0.003\right)$. The elderly patients who think that reimbursement for inpatients is higher than that for outpatients have the highest rate of inappropriate admission (43.9\%). Moreover, the affordability of older patients to hospitalisation costs was significantly associated with inappropriate admission $\left(\chi^{2}=29.52, \mathrm{p}=0.009\right)$. Elderly patients who find it difficult to afford hospitalisation cost had the lowest inappropriate admission rate $(38.3 \%)$. In response to the doctor's admission requirest, the disease severity, and hospitalisation convenience, no statistically significant difference was found in the distribution of admission appropriateness of the elderly.

In the non-elderly group, patients who decided to be admitted to hospitals on their own had a higher inappropriate admission rate than those whose decision was made by someone else $\left(\chi^{2} 214.258, p=0.007\right)$. Patients who think that the reimbursement for inpatients is higher than that for outpatients had the highest rate of inappropriate admission $\left(\chi^{2}=211.542, \mathrm{p}=0.003\right)$, and the results were consistent with those in the elderly group.

\section{Determinants of inappropriate admission to hospital for elderly people}

Logistic regression based on the interaction of age (elderly and non-elderly group) and other factors shows that the interaction between age and gender and between age and chronic disease influenced patients' inappropriate admission to hospital (table 3). Compared with the non-elderly, the risk of inappropriate admission of the female elderly was lower ( $\mathrm{OR}=0.33,95 \%$ CI 0.15 to 0.73 ), and the elderly with chronic diseases were more likely to be inappropriately admitted to hospital (OR=3.33, 95\% CI 1.23 to 9.04).

The results of the binary logistic regression for elderly patients are shown in table 4. Taking Yilong as the reference group, rural elderly patients in Dingyuan had a higher risk of inappropriate admission to hospital ( $\mathrm{OR}=9.74,95 \% \mathrm{CI} 3.30$ to 28.41). A higher age corresponds to a lower possibility of inappropriate admission ( $\mathrm{OR}=0.94,95 \%$ CI 0.90 to 0.99 ). Elderly people in the surgery department are less likely to be admitted to 
Table 2 Distributions of characteristics of appropriateness admission under the subjective perception of patients

Elderly Non-elderly

\begin{tabular}{|c|c|c|c|c|c|c|c|c|}
\hline & & Appropriate & Inappropriate & & & Appropriate & Inappropriate & \\
\hline Variable & $\mathrm{N}$ (column \%) & N (line \%) & N (line \%) & $P$ values & N (column \%) & N (line \%) & N (line \%) & $P$ values \\
\hline \multicolumn{9}{|l|}{ RTDAR } \\
\hline No doubt & $63(27.4)$ & $90(56.3)$ & $70(43.8)$ & 0.119 & $160(37.9)$ & $46(73.0)$ & $17(27.0)$ & 0.007 \\
\hline Doubt & $17(7.4)$ & $16(59.3)$ & $11(40.7)$ & & $27(6.4)$ & $16(94.1)$ & $1(5.9)$ & \\
\hline Expected & $124(53.9)$ & $124(65.3)$ & $66(34.7)$ & & $190(45.0)$ & $84(67.7)$ & 40 (32.3) & \\
\hline $\begin{array}{l}\text { Family } \\
\text { requirements }\end{array}$ & $13(5.7)$ & $8(80.0)$ & $2(20.0)$ & & $10(2.4)$ & $8(61.5)$ & $5(38.5)$ & \\
\hline Others & $13(5.7)$ & $12(34.3)$ & $23(65.7)$ & & $35(8.3)$ & $7(53.8)$ & $6(46.2)$ & \\
\hline \multicolumn{9}{|l|}{ Disease severity } \\
\hline Light & $8(3.5)$ & 39 (58.2) & $28(41.8)$ & 0.546 & $67(15.9)$ & $7(87.5)$ & $1(12.5)$ & 0.697 \\
\hline General & $46(20.0)$ & $63(56.3)$ & $49(43.8)$ & & $112(26.5)$ & $32(69.6)$ & $14(30.4)$ & \\
\hline Serious & $176(76.5)$ & $148(60.9)$ & $95(39.1)$ & & $243(57.6)$ & $122(69.3)$ & $54(30.7)$ & \\
\hline \multicolumn{9}{|c|}{ Comparison of $R_{0}$ and $R_{1}$} \\
\hline $\mathrm{RO}>\mathrm{RI}$ & $62(27.0)$ & $37(80.4)$ & $9(19.6)$ & 0.003 & $46(10.9)$ & $54(87.1)$ & $8(12.9)$ & 0.003 \\
\hline $\mathrm{RI}>\mathrm{RO}$ & $101(43.9)$ & $101(53.2)$ & $89(46.8)$ & & $190(45.0)$ & $64(56.1)$ & $37(43.9)$ & \\
\hline Unknown & $67(29.1)$ & $112(60.2)$ & 74 (39.8) & & $186(44.1)$ & $43(70.9)$ & $24(29.1)$ & \\
\hline \multicolumn{9}{|l|}{ Affordability } \\
\hline Difficult & $73(31.7)$ & $37(61.7)$ & $23(38.3)$ & 0.009 & $60(14.2)$ & $61(83.6)$ & $12(16.4)$ & 0.842 \\
\hline Reluctantly & $83(36.1)$ & $124(57.9)$ & $90(42.1)$ & & $214(50.7)$ & $54(65.1)$ & 29 (34.9) & \\
\hline Fully payable & $74(32.2)$ & $89(60.1)$ & 59 (39.9) & & 148 (35.1) & $46(62.2)$ & 28 (37.8) & \\
\hline \multicolumn{9}{|c|}{ Hospitalisation convenience } \\
\hline Yes & $116(50.4)$ & $169(60.8)$ & 109 (39.2) & 0.085 & 278 (65.9) & $75(64.7)$ & 41 (35.3) & 0.368 \\
\hline No & $114(49.6)$ & $81(56.3)$ & $63(43.8)$ & & $144(34.1)$ & $86(75.4)$ & $28(24.6)$ & \\
\hline
\end{tabular}

$\mathrm{RI}$, reimbursement of inpatient; RO, reimbursement of outpatient; RTDAR, response to doctor's admission requirement.

hospital inappropriately $(\mathrm{OR}=0.21,95 \% \mathrm{CI} 0.07$ to 0.61$)$. For the subjective cognition of elderly patients, our study showed that only patients who have doubts about the doctor's admission requirement but decided to be hospitalised after communication with the doctor were the least likely to be admitted inappropriately $(\mathrm{OR}=0.07$, $95 \%$ CI 0.01 to 0.76 ).

\section{DISCUSSION}

By descriptive analysis and logistic regression analysis, we studied the characteristics and determinants of inappropriate admission of elderly people in rural China. Our results will help us determine the reasons for inappropriate admission of elderly people in rural China from the perspective of supply and demand by discussing the determinant factors. Our study would also try to give some suggestions to the Chinese government for controlling inappropriate admission of the elderly, and provide new observations for researchers in this field.

\section{Inappropriate admission of the elderly in rural China}

In this study, the inappropriate admission rate of patients aged over 60years in rural areas in China is 30\%, which is generally higher than the $27 \%$ reported in the $\mathrm{UK}^{18}{ }^{18} 9.8 \%$ in Italy, $^{14} 9.2 \%$ in Switzerland ${ }^{19}$ and $8.8 \%$ in France. ${ }^{13}$ Zhang $e t a t^{20}$ found that the inappropriate admission rate of patients aged over 60 years in rural areas in China was $14.3 \%$, which was also lower than the results of this study. This result may be attributed to the differences among the studies with regards to the health services system, geographical environment or study period. These studies were mainly concerned with elderly people of different age groups and in different admission departments. The inappropriate admission rate of elderly patients (30\%) was lower than that of non-elderly patients (40.8\%) probably because the body functions of elderly patients are worse than those of non-elderly patients. Moreover, an elderly person presents more obvious and serious symptoms at the onset of a disease and is thus less likely to be inappropriately admitted.

\section{Characteristics of elderly patients who were hospitalised inappropriately}

Significant differences were found in the distribution of inappropriate admission among elderly patients across different counties. Among the four surveyed areas, 


\begin{tabular}{|c|c|c|c|c|c|}
\hline Variable & COR $(95 \% \mathrm{Cl})$ & AOR $(95 \% \mathrm{Cl})^{*}$ & Variable & COR $(95 \% \mathrm{Cl})$ & AOR $(95 \% \mathrm{Cl})^{*}$ \\
\hline County (ref: Yilong)†‡ & & & Health status (ref: good) & & \\
\hline Dingyuan & 4.03 (2.52 to 6.43$)$ & 5.99 (3.08 to 11.68$)$ & Poor & 0.57 (0.27 to 1.22$)$ & 0.83 (0.33 to 2.06$)$ \\
\hline Weiyuan & 1.16 (0.99 to 2.61$)$ & 1.85 (0.98 to 3.48$)$ & RTDAR (ref: no doubt)† & & \\
\hline $\begin{array}{l}\text { Disease category } \\
\text { (ref: circulatory } \\
\text { disease)† }\end{array}$ & & & Family requirements & 0.68 (0.27 to 1.73$)$ & 0.73 (0.25 to 2.09$)$ \\
\hline Respiratory disease & $1.02(0.61$ to 1.71$)$ & $0.83(0.43$ to 1.58$)$ & Others & 2.39 (1.26 to 4.52$)$ & $1.91(0.89$ to 4.09$)$ \\
\hline Digestive disease & 1.46 (0.86 to 2.48$)$ & 2.84 (1.44 to 5.58$)$ & Disease severity (ref: light) & & \\
\hline Bones and muscles & 2.08 (0.87 to 4.98$)$ & 1.45 (0.52 to 4.06$)$ & $\begin{array}{l}\text { Comparison of } \mathrm{RO} \text { and } \\
\mathrm{RI}(\text { ref: } \mathrm{RO}>\mathrm{RI}) \dagger\end{array}$ & & \\
\hline Injury and poisoning & 0.20 (0.06 to 0.67$)$ & 0.99 (0.24 to 4.13$)$ & $\mathrm{Rl}<\mathrm{RO}$ & 4.09 (2.32 to 7.21$)$ & $2.13(1.08$ to 4.21$)$ \\
\hline Symptoms and signs & 1.33 (0.81 to 2.18$)$ & 1.63 (0.91 to 2.93$)$ & Unknown & 3.38 (1.90 to 6.02 ) & $1.62(0.80$ to 3.27$)$ \\
\hline Others & 1.57 (0.90 to 2.73$)$ & 1.30 (0.64 to 2.64$)$ & Affordability (ref: difficult)†‡ & & \\
\hline $\begin{array}{l}\text { Department } \\
\text { (ref: internal } \\
\text { medicine)†‡ }\end{array}$ & & & Reluctantly & 1.87 (1.19 to 2.94$)$ & 1.14 (0.66 to 1.96$)$ \\
\hline Surgery & 0.32 (0.17 to 0.58$)$ & $0.11(0.05$ to 0.25$)$ & Fully payable & 1.80 (1.13 to 2.89$)$ & $1.84(1.05$ to 3.24$)$ \\
\hline Paediatric & 3.28 (2.20 to 4.88 ) & 4.26 (1.94 to 9.35$)$ & $\begin{array}{l}\text { Hospitalisation convenience } \\
\text { (ref: yes) }\end{array}$ & 0.89 (0.64 to 1.23$)$ & 1.25 (0.84 to 1.88$)$ \\
\hline
\end{tabular}

${ }^{*}$ Adjusted for all other covariates listed in the table.

†Univariate regression analysis $\mathrm{p}<0.05$.

łLogistic regression analysis $p<0.05$.

AOR, adjusted OR; COR, crude OR; DOCD, diagnosis of chronic disease; RI, reimbursement of inpatient; RO, reimbursement of outpatient;

RTDAR, response to doctor's admission request.

Dingyuan had the highest rate of inappropriate admission, whereas Yilong had the lowest. This could be due to the differences in health policies, geographical environments and customs between the counties. Elderly patients who could afford hospitalisation costs with difficulty had the lowest inappropriate admission rate. This could be due to the economic difficulties that restrict utilisation of health services for elderly patients in rural areas in China. ${ }^{21}$ Our research proves that elderly people who have a correct understanding of medical insurance reimbursement have higher inappropriate admission rates, probably because the medical insurance reimbursement policy has promoted the demand for excessive release of hospitalisation services for elderly patients. ${ }^{22}$

\section{Determinants of inappropriate admission for elderly people}

Gender and chronic diseases are the specific factors affecting the inappropriate admission of elderly people. Compared with non-elderly patients, the tendency of female elderly patients for inappropriate hospitalisation was less obvious. Moreover, the risk of inappropriate admission of elderly patients with chronic diseases was higher, which is consistent with the findings of Gamper, probably because relative to non-elderly people, elderly people are less aware of the disease. Moreover, perfect chronic disease management is lacking in rural areas in China. When the symptoms of a chronic disease appear, it is easier for the elderly to misjudge the disease early and blindly choose to be admitted to a hospital.

Logistic regression shows that the risk of inappropriate admission varies across different counties in China's rural areas. The health policy in different counties, especially the medical insurance policy, plays a key role in the inappropriate admission of elderly patients (the New Rural Cooperative Medical system [NRCMS] reimbursement policies in the survey areas in 2017 are shown in online additional file 2). A large gap exists between 


\begin{tabular}{|c|c|c|}
\hline Variable & COR (95\% Cl) & AOR $(95 \% \mathrm{Cl})^{*}$ \\
\hline \multicolumn{3}{|l|}{ County (ref: Yilong)† } \\
\hline Huining & 1.60 (0.63 to 4.06$)$ & 0.67 (0.21 to 2.07$)$ \\
\hline Dingyuan & $6.33(2.63$ to 15.25$)$ & 9.74 (3.30 to 28.41$)$ \\
\hline Weiyuan & 3.21 (1.29 to 7.98$)$ & 3.36 (1.08 to 10.46$)$ \\
\hline Age (years)† & 0.99 (0.95 to 1.03$)$ & 0.94 (0.90 to 0.99$)$ \\
\hline Gender (ref: male)† & $0.64(0.36$ to 1.14$)$ & $0.49(0.25$ to 2.98$)$ \\
\hline \multicolumn{3}{|c|}{ Department (ref: internal medicine)† } \\
\hline Surgery & $0.47(0.18$ to 1.20$)$ & 0.21 (0.07 to 0.61$)$ \\
\hline Others & 1.65 (0.55 to 4.96$)$ & $1.49(0.35$ to 6.27$)$ \\
\hline \multicolumn{3}{|l|}{ RTDAR (ref: no doubt)† } \\
\hline Doubt & 0.17 (0.02 to 1.38$)$ & 0.07 (0.01 to 0.76$)$ \\
\hline Expected & 1.29 (0.66 to 2.52$)$ & 0.87 (0.39 to 1.96$)$ \\
\hline Family requirements & 1.69 (0.49 to 5.89$)$ & $1.48(0.34$ to 6.34$)$ \\
\hline Others & 2.32 (0.68 to 7.89$)$ & $4.14(0.94$ to 18.24$)$ \\
\hline \multicolumn{3}{|c|}{ Affordability (ref: difficult)‡ } \\
\hline Reluctantly & 2.73 (1.27 to 5.87$)$ & 1.69 (0.68 to 4.20$)$ \\
\hline Complete & 3.09 (1.42 to 6.73$)$ & 2.84 (1.13 to 7.17$)$ \\
\hline
\end{tabular}

Note, forward stepwise: $\alpha_{\text {entry }}=0.1, \alpha_{\text {removal }}=0.15$

*Logistic regression analysis $\mathrm{p}<0.05$

†Adjusted for all other covariates listed in the table.

$\ddagger$ Univariate regression analysis $p<0.05$.

AOR, adjusted OR; COR, crude OR; RTDAR, response to doctor's admission request.

outpatient and hospitalisation compensation in the survey area. Especially at county-level hospitals, the general outpatient reimbursement is not covered, hence, elderly people in rural areas prefer hospitalisation to get more medical expenses reimbursement. Dingyuan has the highest risk of inappropriate admission for elderly patients, which may be caused by its high level of reimbursement policy by the NRCMS. In 2017, the reimbursement ratio of NRCMS in Dingyuan county-level hospitals was $85 \%$, and the annual payment limitation of each inpatient could reach $¥ 200000$, which was higher than that of Yilong and Weiyuan. Although the reimbursement ratio of NRCMS and the annual payment limitation of each inpatient in Yilong are higher than that of Weiyuan, the risk of inappropriate admission of elderly patients in Weiyuan was higher, maybe because of the greater impact of its geographical environment. Yilong is located in low hills and has a convenient mode of transportation, whereas Weiyuan is located in a high mountainous area, where the terrain is complex and the traffic is inconvenient, which makes it more difficult for elderly patients to travel and makes them choose to be admitted for convenience of treatment. ${ }^{23}$

Gender and age also had an impact on the inappropriate admission of elderly people in rural areas in China. Compared with female elderly patients, the tendency for inappropriate admission of male elderly patients is more obvious. This observation is probably because men generally enjoy a higher status in the family and society ${ }^{24}$ and the health condition of men receives more attention, making them willing to undergo hospitalisation to ensure treatment, resulting in inappropriate admission. With increasing age, elderly patients are less likely to be admitted inappropriately. This result is consistent with that of other studies. ${ }^{25}$ The reason is mainly because older patients have severely reduced body functions, and their diseases are more serious; hence, inappropriate admission is relatively rare.

The department was also a key factor in the inappropriate admission of elderly patients. Compared with surgery, medical patients are more prone to inappropriate admissions (the inappropriate admission rate is $31.3 \%$ ), which is consistent with the results of Ferrero. ${ }^{26}$ This observation might be caused by two reasons: On the one hand, many surgery-related indicators are included in the original evaluation indicators in $\mathrm{AEP},{ }^{27}$ which improves the rationality of surgical patient admission. On the other hand, most elderly patients suffer from chronic diseases, making it easy to select internal medicine departments for treatment. However, the performance appraisal of Chinese hospitals is always directly linked to departmental income. The internal medicine income is significantly lower than that of surgery, which causes physicians to produce more induced demand in pursuit of departmental income. ${ }^{28}$ In addition, the hospital lacks admission standards for the severity of the disease, and the intensity of medical services required. These factors have increased the possibility of inappropriate admission to the elderly.

The response of rural elderly patients to hospital admission requirements directly affects the appropriateness of admission. Elderly patients who have doubts about the doctor's admission requirements and have communicated to the doctor before deciding to enter the hospital have significantly less possibility of being admitted inappropriately. The influence of this factor on the appropriateness of hospitalisation in the rural elderly is mainly reflected in two points. First, Chinese rural areas have not established a perfect 'family doctor first' consultation mechanism, and rural elderly patients lack the guidance of the first diagnosis and the correct understanding of their disease. Cultural degree is low especially for the elderly patients in rural areas in China, which makes their judgement of the disease entirely dependent on the clinician. For doctors' admission requirements, if elderly patients communicate with doctors effectively, understand their own disease condition and make admission decisions accordingly, the tendency of inappropriate admission of elderly patients is significantly reduced. Second, the doctor's request for admission of the patient is based on the judgement of the patient's basic condition and with regard to factors of medical accident risk aversion, departmental income, and so on. ${ }^{29}{ }^{30}$ Proved by Aida Bianco and Mytton, doctors' clinical decision-making has become a key point affecting the appropriateness of patients' admission. 


\section{CONCLUSION}

This study found that the rate of inappropriate admission of elderly patients in county-level hospitals from rural China was high. We found that chronic disease was the specific factor, and county, department and response to the doctor's admission requirements were the determinants of inappropriate admission of elderly patients. We believe that these factors are closely related to the management of chronic diseases in the elderly, regional health policies (especially the NRCMS), department management and family doctor service in rural China. Therefore, this study suggests that the government can control the inappropriate admission of the elderly from the following points. (1) The NRCMS management department should adjust the reimbursement ratio of hospitalisation and outpatients according to actual inspections and increase the ratio of outpatient reimbursement to a certain extent, reducing unnecessary hospitalisation needs. (2) Hospital departments need to establish a performance appraisal system with medical quality and patient satisfaction as the core, and admission criteria for the severity of the disease and the intensity of required medical services should also be established. (3) To improve elderly patients' cognition of the disease, China's rural areas need to establish a family doctor service based on the concept of health management, ${ }^{31}$ and provide medical consultation and guidance for elderly patients.

\section{LIMITATION}

This study has two limitations. First, the cross-sectional study design could not determine the causal relationship between unreasonable admission and related factors in elderly patients. Second, no unified identification standard exists for the inappropriate admission of elderly patients in the current studies, ${ }^{32}$ making the identification of inappropriate admission dependent on AEP. The AEP applicable to the entire population may not be the best tool for evaluating the inappropriate admission of elderly patients. ${ }^{1733-35}$

Contributors $\mathrm{XH}, \mathrm{HG}$ and $\mathrm{YZ}$ participated in the conception and design of the manuscript. XH and $H G$ contributed equally to the development of this research study. YZ, HL, DS, JC, SL and DJ participated in the data collection and statistical analysis. YC helped draft, review and revise the manuscript. All authors gave their approval to publish this version of the manuscript.

Funding This research is supportedby the National Natural Science Foundation of China (71473096).

Disclaimer This work has never been published or presented at any time in the past.

\section{Competing interests None declared.}

Patient consent for publication Not required.

Ethics approval The research methods and investigation tools in this study were approved by the Ethics Committee of Tongji Medical College, Huazhong University of Science and Technology (IORG No: IORG0003571).

Provenance and peer review Not commissioned; externally peer reviewed.

Data sharing statement The anonymised data set can be requested by sending an email to the corresponding author.
Open access This is an open access article distributed in accordance with the Creative Commons Attribution Non Commercial (CC BY-NC 4.0) license, which permits others to distribute, remix, adapt, build upon this work non-commercially, and license their derivative works on different terms, provided the original work is properly cited, appropriate credit is given, any changes made indicated, and the use is non-commercial. See: http://creativecommons.org/licenses/by-nc/4.0/.

\section{REFERENCES}

1. National Bureau of Statistics of China. Statistical Communiqué of the People's Republic of China on the 2017 National Economic and Social Development [N]. China Information Daily, 2018-03-01 (003).

2. Center for Health Statistics and Information. An analysis report of national health services survey in china, 2013 [M]. Peking Union Medical College Press 2015.

3. Gao X, Chen Y, Chu Z, et al. Analysis of characteristic excessive demand for elderly inpatient service and causes covered by NRCMS [J]. Chinese Journal of Hospital Administration 2015:279-82.

4. Lavis JN, Anderson GM. Appropriateness in health care delivery: definitions, measurement and policy implications. CMAJ 1996;154:321-8.

5. Payne SM. Identifying and managing inappropriate hospital utilization: a policy synthesis. Health Serv Res 1987;22:709-69.

6. Campbell J. Inappropriate admissions: thoughts of patients and referring doctors. J R Soc Med 2001;94:628-31.

7. World Health Organization. China country assessment report on ageing and health $[R, 2016$.

8. Chen J. Research on Demand an Utilization of Health Service among the Elderly in Rural Areas under the Background of Ageing[D] Peking Union Medical College, 2012.

9. Chun-yan XIE, Shan-lian HU, Guo-zhen SUN. et al. Exploration and experience of provider payment system reform in china [J]. Chinese Health Economics 2010;29:27-9.

10. Creditor MC. Hazards of hospitalization of the elderly. Ann Intern Med 1993;118:219-23.

11. Fusco M, Buja A, Piergentili P, et al. Individual and hospital-related determinants of potentially inappropriate admissions emerging from administrative records. Health Policy 2016;120:1304-12.

12. Gamper G, Wiedermann W, Barisonzo R, et al. Inappropriate hospital admission: interaction between patient age and co-morbidity. Intern Emerg Med 2011;6:361-7.

13. Menand E, Lenain E, Lazarovici $\mathrm{C}$, et al. French multicenter evaluation of the appropriateness of admission to the emergency department of the over-80s. J Nutr Health Aging 2015;19:681-7.

14. Bianco A, Pileggi C, Rizza P, et al. An assessment of inappropriate hospital bed utilization by elderly patients in southern Italy. Aging Clin Exp Res 2006;18:249-56.

15. Mytton OT, Oliver D, Mirza N, et al. Avoidable acute hospital admissions in older people[J]. British Journal of Health Care Management(11) 2013;18:597-603.

16. Gertman PM, Restuccia JD. The appropriateness evaluation protocol: a technique for assessing unnecessary days of hospital care. Med Care 1981;19:855-71.

17. Chen YC. Excessive demand for inpatient service in rural areas [M]. Science Press 2014

18. Leah V, Adams J. Assessment of older adults in the emergency department. Nurs Stand 2010;24:42-5.

19. Ingold BB, Yersin B, Wietlisbach V, et al. Characteristics associated with inappropriate hospital use in elderly patients admitted to a general internal medicine service. Aging 2000;12:430-8.

20. Zhang $\mathrm{Y}$, Zhang L, Li H, et al. Determinants of inappropriate admissions in county hospitals in rural china: A cross-sectional study. Int J Environ Res Public Health 2018;15:1050.

21. Fang-wu C, Xu-li Y, Jie LIU. Analysis on the situation of health service utilization and its influencing factors among aged people[J]. Modern Preventive Medicine 2007-3083-5.

22. Hong-Wei H, Wen-jing L, Jia-yi LI. Medical insurance, health Services Utilization and Excessive Demands for Medical Services - The Impact of Medical Insurance on Utilization of Health Service of the elderly [J]. Journal of Shanxi Finance and Economics University 2015;37:14-24

23. Zhang $Y$, Chen $Y$, Zhang $X$, et al. Current level and determinants of inappropriate admissions to township hospitals under the new rural cooperative medical system in China: a cross-sectional study. BMC Health Serv Res 2014;14:649.

24. Yong-xia T, Wei-guo LUO. Investigation on Family Status of Women in Poor Rural Areas - With Tongwei County in Dingxi of Gansu as an Example [J]. Journal of University of Science and Technology Beijing 2014;30:46-55. 
25. Tavakoli N, Hosseini Kasnavieh SM, Yasinzadeh M, et al. Evaluation of appropriate and inappropriate admission and hospitalization days according to Appropriateness Evaluation Protocol (AEP). Arch Iran Med 2015:18:430-4.

26. Ferrero Benéitez OL, Sánchez Muñoz LA, Corredera Rodríguez C, et al. [Inappropriate admissions to the Department of Internal Medicine evaluated by the AEP (Appropriateness Evaluation Protocol)]. An Med Interna 1998;15:674-5.

27. Mozes B, Schiff E, Modan B. Factors affecting inappropriate hospital stay. Qual Assur Health Care 1991;3:211-7.

28. Gan $\mathrm{CH}$, Zhou X, Zheng RG. Imperfect information, supplier-induced demand and quality of health service[J]. Journal of Finance \& Economics 2007.

29. Yip WC, Hsiao W, Meng Q, et al. Realignment of incentives for health-care providers in China. Lancet 2010;375:1120-30.

30. Osinaike BB, Olusanya O. Inappropriate Intensive Care Unit admissions: Nigerian doctors' perception and attitude. Niger J Clin Pract 2016;19:721-4.
31. Purdy S, Griffin T. Reducing hospital admissions. BMJ 2008;336:4-5.

32. Thwaites R, Glasby J, le Mesurier N, et al. Room for one more? A review of the literature on 'inappropriate' admissions to hospital for older people in the English NHS. Health Soc Care Community 2017;25:1-10.

33. Sánchez-García S, Juárez-Cedillo T, Mould-Quevedo JF, et al. The hospital appropriateness evaluation protocol in elderly patients: a technique to evaluate admission and hospital stay. Scand J Caring Sci 2008;22:306-13.

34. Cardona-Morrell M, Kim JCH, Brabrand M, et al. What is inappropriate hospital use for elderly people near the end of life? A systematic review. Eur J Intern Med 2017;42:39-50.

35. Liu W, Yuan S, Wei F, et al. Reliability and validity of the chinese version appropriateness evaluation protocol. PLoS One 2015;10:e0136498. 